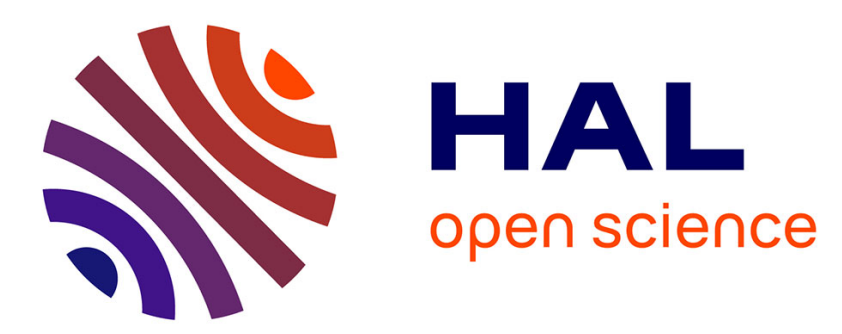

\title{
Comparison between explicit and implicit discrete-time implementations of sliding-mode controllers
}

\author{
Olivier Huber, Vincent Acary, Bernard Brogliato
}

\section{To cite this version:}

Olivier Huber, Vincent Acary, Bernard Brogliato. Comparison between explicit and implicit discretetime implementations of sliding-mode controllers. CDC 2013 - 52nd IEEE Conference on Decision and Control, Dec 2013, Florence, Italy. pp.2870-2875, 10.1109/CDC.2013.6760319 . hal-00870281

\section{HAL Id: hal-00870281 \\ https://hal.inria.fr/hal-00870281}

Submitted on 6 Oct 2013

HAL is a multi-disciplinary open access archive for the deposit and dissemination of scientific research documents, whether they are published or not. The documents may come from teaching and research institutions in France or abroad, or from public or private research centers.
L'archive ouverte pluridisciplinaire HAL, est destinée au dépôt et à la diffusion de documents scientifiques de niveau recherche, publiés ou non, émanant des établissements d'enseignement et de recherche français ou étrangers, des laboratoires publics ou privés. 


\title{
Comparison between explicit and implicit discrete-time implementations of sliding-mode controllers
}

\author{
Olivier Huber, Vincent Acary and Bernard Brogliato
}

\begin{abstract}
Different time-discretization methods for slidingmode control (SMC) are presented. A new discrete-time slidingmode control scheme is proposed for linear time-invariant (LTI) systems. It is error-free in the discretization of the equivalent part of the control input. Results from simulations using the various discretized SMC schemes are shown, with and without perturbations. They illustrate the different behaviours that can be observed.
\end{abstract}

\section{INTRODUCTION}

The time discretization of sliding-mode controllers has witnessed an intense activity in the past 30 years [1]-[6]. This concerns in particular the classical Equivalent-ControlBased Sliding-Mode Control (ECB-SMC), which consists of two sub-controllers: the state-continuous equivalent control $u^{e q}$ and the state-discontinuous control $u^{s}$. In this past research effort, most of the focus was on the discontinuous part of the control, since it introduces numerical chattering. Several solutions to alleviate numerical chattering (that is solely due to the time discretization [7]-[11]) have been proposed [1]-[6], [12], [13], most of them consisting in the definition of a so-called quasi-sliding surface [5] and an explicit discretization of $u^{s}$. The works in [2] and [6] depart from these discrete-time SMC and propose an algorithm which allows the switching variable to take exactly the zero value at sampling times. They are however limited to first order, scalar systems and require some stringent assumptions. Recently a new approach, which may be seen as a (nontrivial) extension of the controllers in [2] and [6], has been proposed in [10], [11]. The basic idea is to implement the discontinuous input $u^{s}$ in an implicit form, while keeping its causality. Then the input has to be computed at each sampling time as the solution to a generalized, set-valued equation, which takes the form of a simple projection on an interval in the simplest cases. This will be recalled later in this paper.

To the best of our knowledge, very few has been done about the discretization of the equivalent part. In this work, we present a study of the effects of discretization on both the equivalent part of the control and the discontinuous part. After presenting the different discretization methods, we propose a new discrete-time control scheme, where the equivalent part is not discretized but rather designed. We

The authors acknowledge the support of the ANR grant CHASLIM (ANR-11-BS03-0007)

The authors are with INRIA Grenoble Rhône-Alpes, BIPOP project-team, 655 avenue de l'Europe, Inovallée, 38334 Saint-Ismier; \{vincent.acary, bernard.brogliato\} @inria.fr, olivier.hubereinria.fr consider systems in the form:

$$
\left\{\begin{array}{l}
\dot{x}(t)=A x(t)+B u(t)+B \xi(t), \\
u(t)=u^{e q}(t)+u^{s}(t), \\
\sigma(t):=C x(t), \\
u^{s}(t) \in-\alpha \operatorname{Sgn}(\sigma(t)),
\end{array}\right.
$$

with $x(t) \in \mathbb{R}^{n}, u(t) \in \mathbb{R}^{p}, \sigma(t) \in \mathbb{R}^{p}, C \in \mathbb{R}^{p \times n}$, and $\alpha>0$. The function $\sigma$ is called the sliding variable and $\mathrm{Sgn}$ is formally introduced in Definition 1. The perturbation $\xi$ is supposed to be at least continuous: noise is not considered in this paper. When $\xi=0$, the system is said to be nominal. The method used to discretize the dynamics is called Zero-Order Hold (ZOH), also known as exact sampled-data representation. It is often considered for technological reasons, but also since there is no error with this discretization method.

In the remainder of this section, we introduce the notation. In Section II we briefly recall the ECB-SMC theory. Then some classical discretization methods are presented in Section III. After we introduce our new discrete-time SMC scheme in Section IV. Simulation results using different time-discretization methods are shown in Sections V and VI, to illustrate the possible different behaviours of the closedloop system.

Let $\mathbf{x}: \mathbb{R}_{+} \times \mathbb{R}^{p} \rightarrow \mathbb{R}^{n}$ be the solution of system (1). Let $x:=\mathbf{x}(\cdot, u)$ be the solution associated with a continuoustime control $u$ and $\bar{x}:=\mathbf{x}(\cdot, \bar{u})$ the solution with a step function $\bar{u}$. Let $\bar{\sigma}:=C \bar{x}$ be the sliding variable in the latter case. The control values change at predefined time instants $t_{k}$, defined as for all $k \in \mathbb{N}, t_{k}:=t_{0}+k h, t_{0}, h \in \mathbb{R}_{+}$. The scalar $h$ is called the timestep. Let $\bar{x}_{k}:=\bar{x}\left(t_{k}\right)$ and $\bar{\sigma}_{k}:=$ $\bar{\sigma}\left(t_{k}\right)$ for all $k \in \mathbb{N}$. Let sgn be the classical single-valued sign function: for all $x>0, \operatorname{sgn}(x)=1, \operatorname{sgn}(-x)=-1$ and $\operatorname{sgn}(0)=0$.

Definition 1 (Multivalued sign function). Let $x \in \mathbb{R}$. The multivalued sign function Sgn: $\mathbb{R} \rightrightarrows \mathbb{R}$ is defined as:

$$
\operatorname{Sgn}(x)= \begin{cases}1 & x>0 \\ -1 & x<0 \\ {[-1,1]} & x=0 .\end{cases}
$$

If $x \in \mathbb{R}^{n}$, then the multivalued sign function Sgn: $\mathbb{R}^{n} \rightrightarrows$ $\mathbb{R}^{n}$ is defined as: $\operatorname{Sgn}(x):=\left(\operatorname{Sgn}\left(x_{1}\right), \ldots, \operatorname{Sgn}\left(x_{n}\right)\right)^{T}$.

\section{THE EQUIVALENT-BASED CONTINUOUS-TIME SLIDING-MODE CONTROLLER}

Let us assume that the triplet $(A, B, C)$ has a strict vector relative degree $(1,1, \ldots, 1)$. This implies that the decoupling 
matrix $C B$ is full rank. The dynamics of the sliding variable in the nominal system (1) (that is with $\xi(t)=0$ ) is

$$
\dot{\sigma}(t)=C A x(t)+C B u^{e q}(t)+C B u^{s}(t) .
$$

The control law $u^{e q}$ is designed such that the system stays on the sliding surface once it has been reached:

$$
\dot{\sigma}(t)=0 \Rightarrow u^{e q}(t)=-(C B)^{-1} C A x(t) .
$$

Then the nominal system (1) can be rewritten as

$$
\dot{x}(t)=\left(I-B(C B)^{-1} C\right) A x(t)+B u^{s}(t),
$$

or equivalently

$$
\dot{x}(t)=\Pi A x(t)+B u^{s}(t),
$$

with $\Pi:=I-B(C B)^{-1} C$. The sliding variable dynamics with the equivalent control is

$$
\left\{\begin{aligned}
\dot{\sigma}(t) & =C B u^{s}(t) \\
u^{s}(t) & \in-\alpha \operatorname{Sgn}(\sigma(t))
\end{aligned}\right.
$$

Two interesting properties of $\Pi$ are $C \Pi=0$ and $\Pi$ is a projector [14]. Taking the integral form of system (6) yields the relation

$$
x(t)=\Phi\left(t, t_{0}\right) x\left(t_{0}\right)+\int_{t_{0}}^{t} \Phi(t, \tau) B u^{s}(\tau) \mathrm{d} \tau,
$$

with $\Phi\left(t, t_{0}\right)=e^{\Pi A\left(t-t_{0}\right)}$ the state transition matrix. Let us state the following result without proof.

Lemma 1. One has $\dot{\Phi}=\Pi A \Phi, \Phi\left(t_{0}, t_{0}\right)=I$, and $C \Phi=C$.

\section{DISCRETE-TIME CONTROLLERS}

From now on, $\bar{u}^{e q}$ and $\bar{u}^{s}$ are sampled control laws defined as right-continuous step functions:

$$
\begin{gathered}
\bar{u}^{e q}(t)=\sum_{k=0}^{\infty} \bar{u}_{k}^{e q} \Lambda_{k}(t), \quad \bar{u}^{s}(t)=\sum_{k=0}^{\infty} \bar{u}_{k}^{s} \Lambda_{k}(t), \\
\Lambda_{k}(t)= \begin{cases}1 & t \in\left[t_{k}, t_{k+1}\right. \\
0 & \text { otherwise. }\end{cases}
\end{gathered}
$$

The goal of the discretization process is to choose the elements of the sequences $\left\{\bar{u}_{k}^{e q}\right\}$ and $\left\{\bar{u}_{k}^{s}\right\}$ such that the discrete-time system exhibits properties as close as possible to the ones with a continuous-time controller. In continuous time, sliding-mode control systems have their evolution divided into two phases: the reaching phase where $\|\sigma\|>0$ and is decreasing, and the sliding phase where $\sigma=0$ and the sliding motion occurs. It is well know that the sliding motion does not occur in general in discrete time. By analogy with the Filippov's solutions we define the following.

Definition 2 (Discrete-time sliding phase). A system (1), in its sampled-data form, is in the discrete-time sliding phase if $\bar{u}^{s}$ takes values in $(-\alpha, \alpha)^{p}$.

Such a definition appears to be new in the discrete-time sliding-mode control field since it implies that the discretetime discontinuous controller is itself set-valued, just as its continuous-time counterpart in (1) and (7). This will be made possible with an implicit implementation, as proved in [10] and [11]. It is crucial not to define the sliding phase in terms of $\bar{\sigma}_{k}$, but rather in terms of the discontinuous input $\bar{u}^{s}$.

Integrating the nominal version of (1) over $\left[t_{k}, t_{k+1}\right)$ and using the expressions in (9), we obtain the $\mathrm{ZOH}$ discretization of the system:

$$
\bar{x}_{k+1}=e^{A h} \bar{x}_{k}+B^{*} \bar{u}_{k}^{e q}+B^{*} \bar{u}_{k}^{s},
$$

with $B^{*}:=\int_{t_{k}}^{t_{k+1}} e^{A\left(t_{k+1}-\tau\right)} B \mathrm{~d} \tau$. We now present different choices for the values $\bar{u}_{k}^{e q}$ and $\bar{u}_{k}^{s}$. Firstly standard methods are described, while the new method is studied in the next section. Here $\bar{u}_{k}^{e q}$ and $\bar{u}_{k}^{s}$ are the discretized values of the continuous-time control law $u^{e q}$ and $u^{s}$. From all the possible time-discretization schemes, we focus on the one-step explicit, implicit, and midpoint ones. With the expressions found for $u^{e q}$ and $u^{s}$ in (4) and (7), the proposed discretized values for $\bar{u}_{k}^{e q}$ are

$$
\begin{aligned}
\bar{u}_{k, e}^{e q} & =-(C B)^{-1} C A \bar{x}_{k} & & \text { explicit input } \\
\bar{u}_{k, i}^{e q} & =-(C B)^{-1} C A \bar{x}_{k+1} & & \text { implicit input } \\
\bar{u}_{k, m}^{e q} & =1 / 2\left(\bar{u}_{k, e}^{e q}+\bar{u}_{k, i}^{e q}\right) & & \text { midpoint input, }
\end{aligned}
$$

and the two possibilities for $\bar{u}_{k}^{s}$ are

$$
\begin{array}{ll}
\bar{u}_{k}^{s}=-\alpha \operatorname{sgn}\left(\bar{\sigma}_{k}\right) & \text { explicit input } \\
\bar{u}_{k}^{s} \in-\alpha \operatorname{Sgn}\left(\bar{\sigma}_{k+1}\right) & \text { implicit input. }
\end{array}
$$

The objective in Sections V and VI is to study the behaviour of the closed-loop system when different combinations of equations (12a)-(12c) and (13a)-(13b) are used. The most commonly used control law is the combination of (12a) and (13a). This kind of discretization has been studied in [7], [8], [15], with a focus on the sequence formed by $\bar{\sigma}_{k}$ once the system state approaches the sliding manifold. The implicit discretization (13b) was first introduced in [10] and [11]. With this method, for each $k \in \mathbb{N}, \bar{u}_{k}^{s}$ is computed as the solution to the generalized equation

$$
\left\{\begin{array}{l}
\widetilde{\sigma}_{k+1}=\bar{\sigma}_{k}+C B^{*} \bar{u}_{k}^{s} \\
\bar{u}_{k}^{s} \in-\alpha \operatorname{Sgn}\left(\widetilde{\sigma}_{k+1}\right) .
\end{array}\right.
$$

Let us write the discrete-time system with an implicit discretization of $u^{s}$ and let $\bar{u}_{k}^{e q}$ be computed using a method in equations (12a)-(12c)

$$
\left\{\begin{array}{l}
\bar{x}_{k+1}=e^{A h} \bar{x}_{k}+B^{*} \bar{u}_{k}^{e q}+B^{*} \bar{u}_{k}^{s} \\
\widetilde{\sigma}_{k+1}=C \bar{x}_{k}+C B^{*} \bar{u}_{k}^{s} \\
\bar{u}_{k}^{s} \in-\alpha \operatorname{Sgn}\left(\widetilde{\sigma}_{k+1}\right) .
\end{array}\right.
$$

Nothing guarantees that $C\left(e^{A h} \bar{x}_{k}+B^{*} \bar{u}_{k}^{e q}\right)=C \bar{x}_{k}$. Hence, $\widetilde{\sigma}_{k+1}$ is in general different than $\bar{\sigma}_{k+1}$ because of the discretization error on $u^{e q}$. Therefore, it can be considered as an approximation of $\bar{\sigma}_{k+1}$. With the $\operatorname{Sgn}(\cdot)$ multifunction and $C B^{*}$ positive definite, (14) has a unique solution $\bar{u}_{k}^{s}$, a function of $\bar{\sigma}_{k}$ (hence $\bar{x}_{k}$ ). When the control is scalar or $C B^{*}$ is diagonal, a solution to (14) can be computed as a simple projection: $\bar{u}_{k}^{s}=-\operatorname{proj}_{[-\alpha, \alpha]^{p}}\left(\left(C B^{*}\right)^{-1} \bar{\sigma}_{k}\right)$. 


\section{EXACT DISCRETE EQUIVALENT CONTROL}

Let us propose a new control scheme for a discrete-time LTI plant using sliding-mode control. Its derivation is along the same lines as in Section II, that is we first design the equivalent control $\bar{u}^{e q}$ and then the discontinuous part $\bar{u}^{s}$.

As showed in (7), $u^{e q}$ is defined such that the dynamics of the sliding variable depends only on the input $u^{s}$. Starting from (11) and left multiplying by $C$, one obtains:

$$
C \bar{x}_{k+1}=C e^{A h} \bar{x}_{k}+C B^{*} \bar{u}_{k}^{e q}+C B^{*} \bar{u}_{k}^{s} .
$$

Using (8) with $t=t_{k+1}$ and $t_{0}=t_{k}$, we obtain:

$\sigma\left(t_{k+1}\right)=C \Phi\left(t_{k+1}, t_{k}\right) x\left(t_{k}\right)+C \int_{t_{k}}^{t_{k+1}} \Phi\left(t_{k+1}, \tau\right) B u^{s}(\tau) \mathrm{d} \tau$.

Our goal is to have $C \bar{x}_{k+1}=C x\left(t_{k+1}\right)$ if $x\left(t_{k}\right)=\bar{x}_{k}$ and both $u^{s}$ and $\bar{u}^{s}$ set to 0 . Then setting the last term of (16) and (17) to 0 , the following condition holds:

$$
C \Phi\left(t_{k+1}, t_{k}\right) x\left(t_{k}\right)=C e^{A h} \bar{x}_{k}+C B^{*} \bar{u}_{k}^{e q},
$$

that is

$$
C B^{*} \bar{u}_{k}^{e q}=C\left(I-e^{A h}\right) \bar{x}_{k} .
$$

In [3], this expression for the equivalent control was already derived, when the sliding variable is scalar. In [4], using a deadbeat-like approach, a term similar to (19) can also be found. If we substitute this expression for $\bar{u}_{k}^{e q}$ in (16), then, as expected, we obtain:

$$
\bar{\sigma}_{k+1}=\bar{\sigma}_{k}+C B^{*} \bar{u}_{k}^{s} .
$$

For the design of $\bar{u}^{s}$, let us to choose $\bar{u}_{k}^{s}$ such that $\bar{u}^{s}$ steers $\bar{\sigma}_{k}$ to 0 in finite time. Following the work in [10] and [11], we use an implicit discretization of the continuous-time control law. The discrete-time sliding variable dynamics is given by (20) and $\bar{u}_{k}^{s} \in-\alpha \operatorname{Sgn}\left(\bar{\sigma}_{k+1}\right)$. Inserting (19) in (11), the dynamics of the nominal controlled plant is

$$
\left\{\begin{array}{l}
\bar{x}_{k+1}=\left(e^{A h}+B^{*}\left(C B^{*}\right)^{-1} C\left(I-e^{A h}\right)\right) \bar{x}_{k}+B^{*} \bar{u}_{k}^{s} \\
\bar{\sigma}_{k+1}=\bar{\sigma}_{k}+C B^{*} \bar{u}_{k}^{s} .
\end{array}\right.
$$

Using the framework of generalized (set-valued) equations, the discrete-time sliding variable dynamics is

$$
\left\{\begin{array}{l}
\bar{\sigma}_{k+1}=\bar{\sigma}_{k}+C B^{*} \bar{u}_{k}^{s} \\
\bar{u}_{k}^{s} \in-\alpha \operatorname{Sgn}\left(\bar{\sigma}_{k+1}\right) .
\end{array}\right.
$$

This has the same structure as in (14), although with the important difference that we have here $\widetilde{\sigma}_{k+1}=\bar{\sigma}_{k+1}$. With this scheme the two control inputs are

$$
\left\{\begin{array}{l}
\bar{u}_{k}^{e q}=\left(C B^{*}\right)^{-1} C\left(I-e^{A h}\right) \bar{x}_{k} \\
\bar{u}_{k}^{s} \quad \text { solution of }(21)
\end{array}\right.
$$

This controller is causal since $\bar{u}_{k}^{e q}$ depends only on the model parameters and $\bar{x}_{k}$. Moreover $\bar{u}_{k}^{s}$ is the unique solution to (21) given that $C B^{*}>0$.

\section{Simulations of a 2 Dimensional system}

To illustrate the results obtained with different discretization methods, let us simulate the following controlled system:

$$
\begin{cases}\dot{x}(t)=A x(t)+B \bar{u}(t) & A=\left(\begin{array}{cc}
0 & 1 \\
19 & -2
\end{array}\right), \\
\sigma=C x & B=\left(\begin{array}{l}
0 \\
1
\end{array}\right), C^{T}=\left(\begin{array}{l}
1 \\
1
\end{array}\right) .\end{cases}
$$

The matrix $\mathrm{A}$ has the eigenvalues $\lambda_{1}=3.47$ and $\lambda_{2}=$ -5.47 . The dynamics on the sliding surface is given by $\Pi A$, which has eigenvalues 0 and -1 . In the present Section and the next one, we set $\alpha=1$. The initial state is $(-15,20)^{T}$. The first set of simulations uses a timestep of $0.3 \mathrm{~s}$ for the control and the second one a timestep of $0.03 \mathrm{~s}$. The simulations run for $150 \mathrm{~s}$ and were carried out with the SICONOS software package $[16]^{1}$. The figures were created using Matplotlib [17]. The schemes presented in (12a)-(12c) are used, as well as the two schemes in (13a) and (13b) for the discretization of $u^{s}$, on the $\mathrm{ZOH}$ sampled-data version of the system (23).

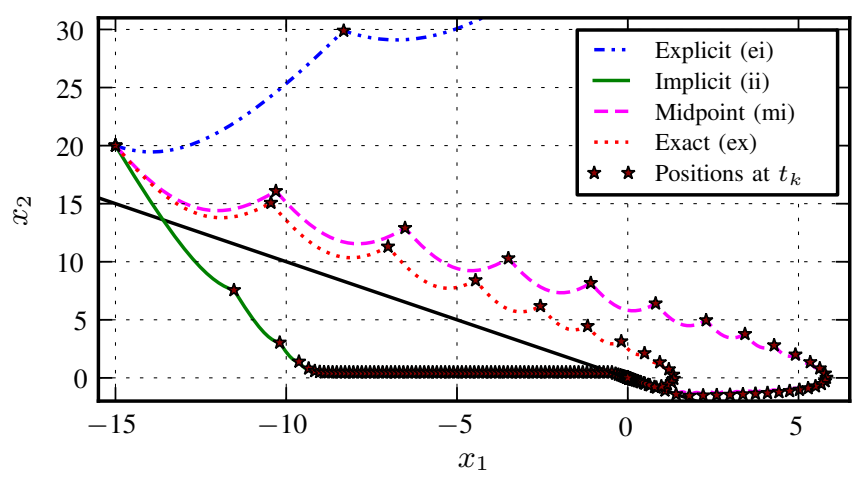

(a) Implicit discretization of $u^{s}$. (ei) is for pair (12a), (13b); (ii) for (12b), (13b); (mi) for (12c), (13b); (ex) for (22).

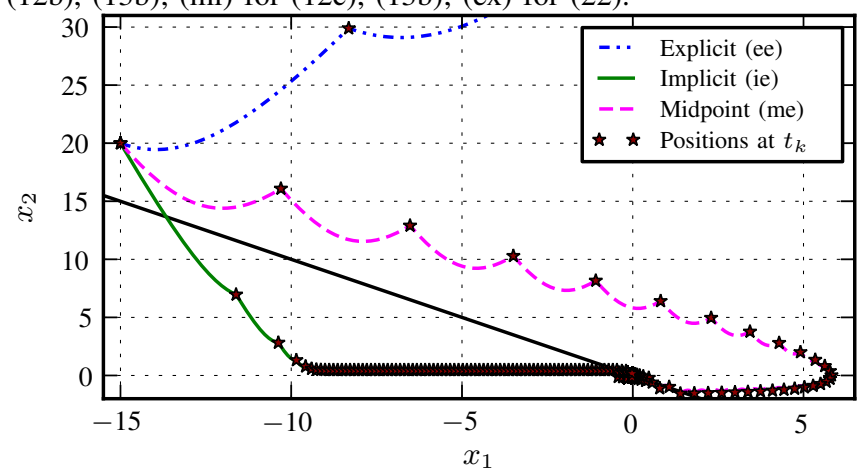

(b) Explicit discretization of $u^{s}$. (ee) is for pair (12a), (13a); (ie) for (12b), (13a); (me) for (12c), (13a).

Fig. 1: Simulations of system (23) using different discretization methods for $u^{e q}$ and with $h=0.3 \mathrm{~s}$.

In Fig. 1 the motion in the reaching phase depends only on discretization method used for the equivalent control $u^{e q}$. It is only near the sliding manifold that the discretization method of the discontinuous control $u^{s}$ plays a role. If the explicit

\footnotetext{
${ }^{1}$ http://siconos.gforge.inria.fr
} 
scheme in (12a) is used for the discretization of $u^{e q}$, the system diverges (Fig. 1, curves (ei) and (ee)). If the implicit scheme in (12b) is used for the discretization of $u^{e q}$, then the discretization error may not affect stability but it can induce some unexpected behaviour. As we can see in Fig. 1, curves (ii) and (ie), the trajectories are crossing the sliding manifold. This phenomenon can be explained by the following fact: let $\Delta \bar{\sigma}_{k}$ be the discretization error on $u^{e q}$ at time $t_{k}$. We have the recursive equation $\bar{\sigma}_{k+1}=\bar{\sigma}_{k}+\Delta \bar{\sigma}_{k}+C B^{*} \bar{u}_{k}^{s}$. Let us consider the implicit discretization of $u^{s}$. If $0<\bar{\sigma}_{k}<C B^{*}$, then the system should enter the discrete-time sliding phase. However if $\Delta \bar{\sigma}_{k}+\bar{\sigma}_{k}<-2 C B^{*}$, then for any value of $\bar{u}_{k}^{s}, \bar{\sigma}_{k+1}<-C B^{*}$. Hence, due to the discretization error, $\bar{u}^{s}$ fails to bring $\bar{\sigma}_{k+1}$ to 0 and the trajectory of the system crosses the sliding manifold. The same happens with the explicit discretization of $u^{s}$. With the midpoint method in (12c), curves (mi) and (me), and with the new control scheme (22), curve (ex), the system reaches the sliding manifold.

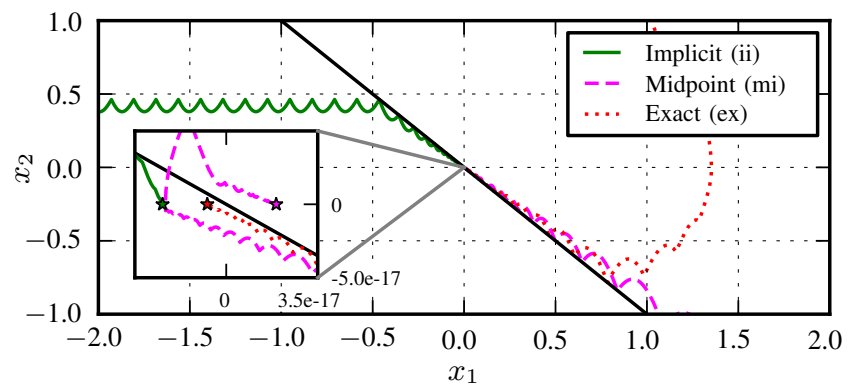

(a) Implicit discretization of $u^{s}$

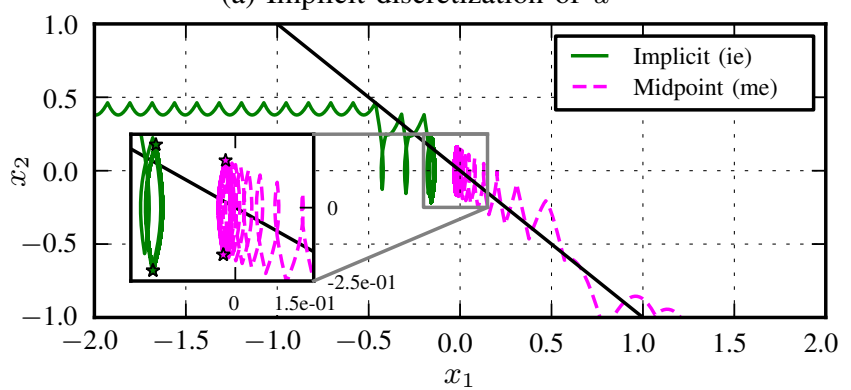

(b) Explicit discretization of $u^{s}$

Fig. 2: Detail of Fig. $1, h=0.3 \mathrm{~s}$.

Near the sliding manifold (Fig. 2a and 2b), the state of the system is more sensitive to the discretization of $u^{s}$. In the implicit case (method (13b), Fig. 2a), in the discrete-time sliding phase, $\bar{\sigma}_{k}$ is very close to $0\left(\bar{\sigma}_{k}=0\right.$ with the exact method). In each case, it converges to a small ball around the origin (its radius is smaller than the machine precision). This is visible on the zoom box in Fig. 2a, where markers indicate the state of the system at each time instant $t_{k}$ during the last second of each simulation. When the explicit method (13a) is used, the system chatters around the sliding manifold, within a neighborhood of order $h$ ( $0.3 \mathrm{~s}$ here), see Fig. $2 \mathrm{~b}$.

In Fig. 3b, $\bar{u}^{s}$ takes its values in $\{-1,1\}$ and starts at some point a finite cycle [8]. This is also visible on the zoom box in Fig. $2 b$ with the help of the markers. In Fig. 3a, for each

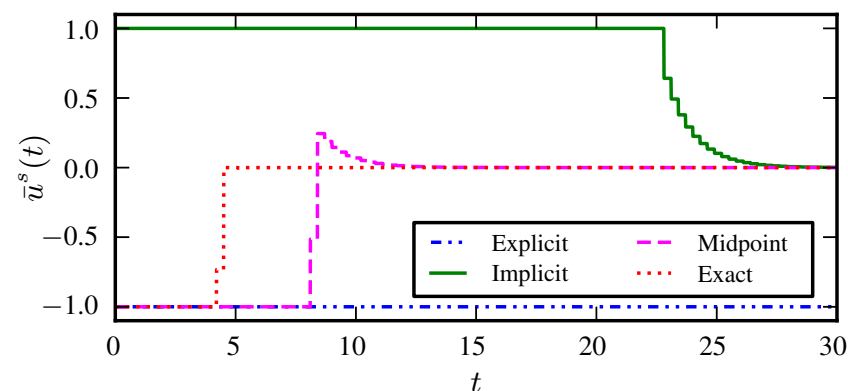

(a) Implicit discretization of $u^{s}$

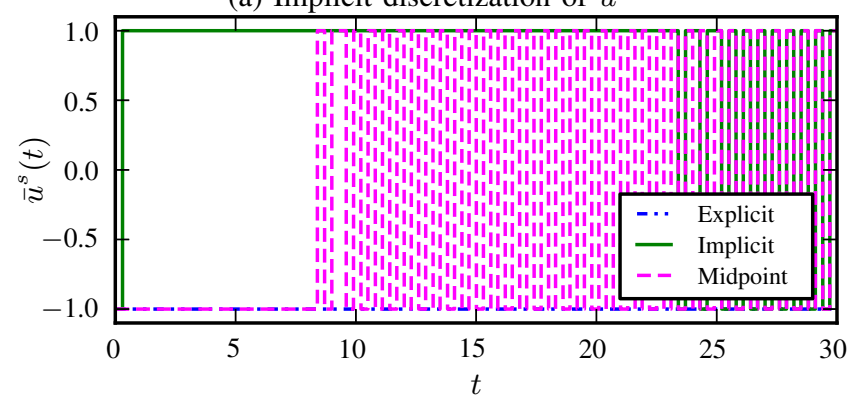

(b) Explicit discretization of $u^{s}$

Fig. 3: Evolution of $\bar{u}^{s}$ for different discretization methods for $u^{e q}, h=0.3 \mathrm{~s}$.

discretization of $u^{e q}, \bar{u}^{s}$ converges to 0 , which is the value that $u^{s}$ takes in the sliding phase. In the implicit and midpoint cases, at the beginning of the discrete-time sliding phase, $\bar{u}^{s}$ takes non zero values since there are discretization errors on $u^{e q}$. That is, if $\bar{\sigma}_{k}=0, \bar{\sigma}_{k+1} \neq 0$. The discontinuous control tries to bring $\bar{\sigma}_{k+1}$ to 0 and counteracts the error. As the states goes to the origin, the error converges to 0 . It can be shown that the error is smaller in the midpoint case as in the implicit case, as illustrated in these simulations. With the exact method of Section IV, $\bar{u}^{s}$ goes to 0 after 1 timestep in the discrete-time sliding phase. In terms of convergence to the sliding manifold, the first closed-loop system to enter the discrete-time sliding phase is the exact method (Fig. 3a), then the midpoint, and the implicit method.

The next set of simulations uses the same parameters as the previous one, except that for timestep which is smaller: $h=0.03 \mathrm{~s}$. In contrast with the results presented in Fig. 1, the closed-loop system is stable is all cases (Fig. 4). The discretization error is smaller and no trajectory crosses the sliding manifold. It is not possible to distinguish the solutions associated with the midpoint and exact methods in Fig. 4 a. In Fig. 5a with the implicit discretization of $u^{s}$, the states converge again to a very small ball near the origin. In the explicit case, there is some numerical chattering, again with the same order of magnitude as the timestep $(h=0.03 \mathrm{~s}$, Fig. 5b). In Fig. 6a, with both the explicit and implicit discretizations in (12a) and (12b), once in the discrete-time sliding phase, $\bar{u}^{s}$ counteracts the discretization error on $u^{e q}$, which is smaller than in Fig. 3a. The discretization error for the midpoint discretization in (12c) is much smaller, and its curve overlaps completely with the one with the exact discretization method. 


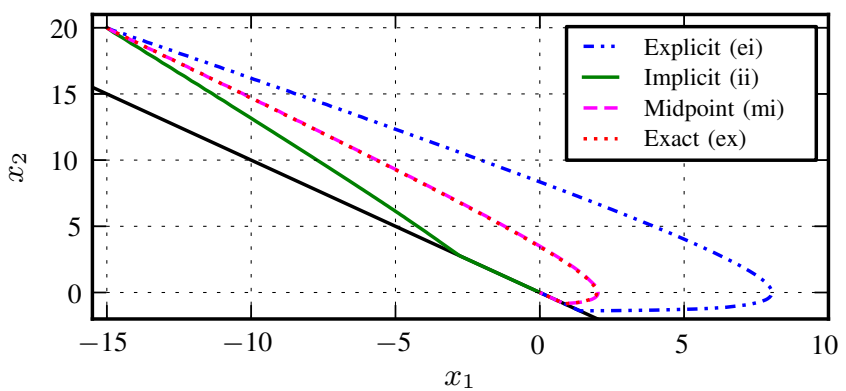

(a) Implicit discretization of $u^{s}$

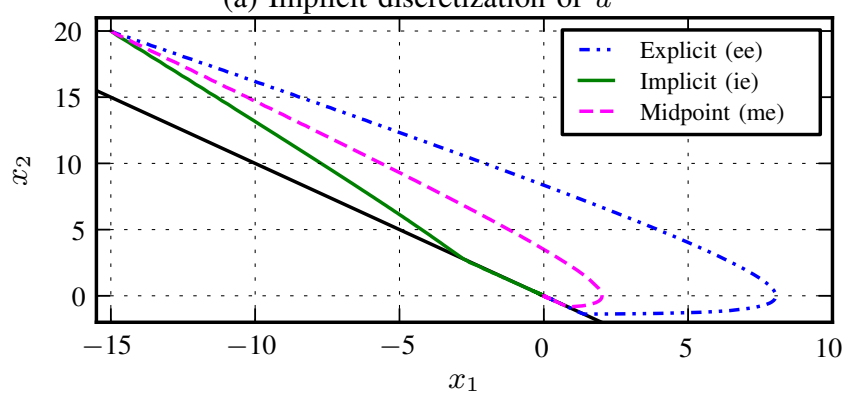

(b) Explicit discretization of $u^{s}$

Fig. 4: Simulations of system (23) using different discretization methods for $u^{e q}$ and with $h=0.03 \mathrm{~s}$

The results presented here bring into view the numerical chattering caused by an explicit discretization of $u^{s}$, while the implicit method is free of it. The importance of the discretization of $u^{e q}$ is also illustrated, with the explicit method leading to a diverging system and the counterintuitive behaviour yielded by the implicit method. The exact method from Section IV produces good results.

\section{PERTURBED CASE}

We now add a perturbation $\xi(t)$ in the system (23). To take it into account, we just need to add a term $p_{k}:=$ $\int_{t_{k}}^{t_{k+1}} e^{A\left(t_{k+1}-\tau\right)} B \xi(\tau) \mathrm{d} \tau$ to the recurrence relation (11). This yields:

$$
\bar{x}_{k+1}=e^{A h} \bar{x}_{k}+B^{*} \bar{u}_{k}^{e q}+B^{*} \bar{u}_{k}^{s}+p_{k} .
$$

In the next set of simulations, the perturbation is $\xi(t)=$ $0.7 \exp \left(\frac{-1}{\left(\bar{u}^{e q}(t)+\bar{u}^{s}(t)\right)^{2}}\right) \sin (2 \pi t)$. Note that $\|\xi(t)\| \leq 0.7$. This particular $\xi$ has been chosen to highlight that with the implicit discretization, $\bar{u}^{s}$ goes to 0 , whereas in the explicit case, $\bar{u}^{s}$ continues to switch between -1 and 1 . With the implicit discretization of $u^{s}$ (Fig. 8a) the closedloop system enters the discrete-time sliding phase at some point. Then it takes such values to counteract the effect of the perturbation, hence imitating the continuous-time Filippov solutions. However the trajectories are now clearly only in a neighborhood of the sliding manifold. Finally in each case in Fig. 8a, $\bar{u}_{k}^{s}$ settles to 0 , as in continuous time. Indeed, with the perturbation $\xi$ used in this simulation, it goes to 0 exponentially fast with respect to the control inputs. On the other hand, with an explicit discretization of $u^{s}$ (Fig. 8b), it is much harder to witness the influence of the perturbation on $\bar{u}^{s}$ since filtering would be necessary to see the effect.

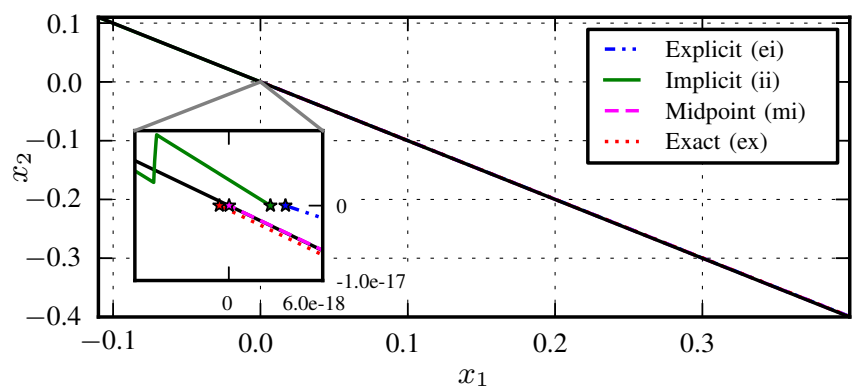

(a) Implicit discretization of $u^{s}$

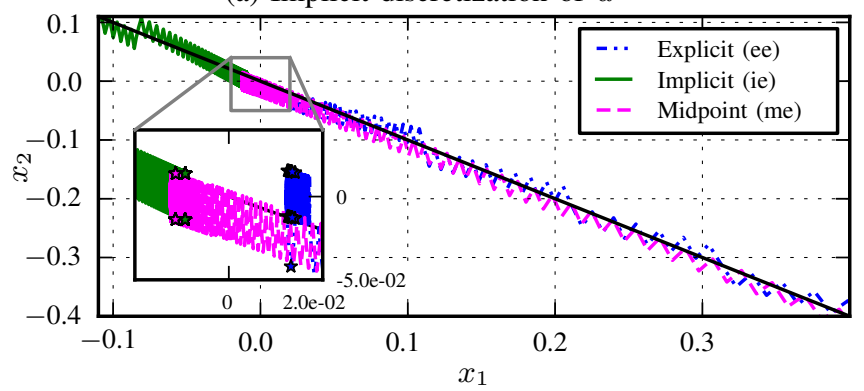

(b) Explicit discretization of $u^{s}$

Fig. 5: Detail of Fig. $4, h=0.03$ s.

\section{CONCLUSION}

In this note a new discrete-time sliding-mode control scheme is proposed, which is error-free in the discretization of the controller $u^{e q}$. This, along with several time discretizations of the classical ECB-SMC method, are analysed from the point of view of their ability to alleviate or suppress the numerical chattering. The analysis is essentially led from numerical simulations obtained with the INRIA software package SICONOS. In particular the influence of the discretization method of the state-continuous equivalent controller is studied, as well as the one of the discontinuous part of the input (explicit versus implicit discretizations). The nominal and perturbed cases are considered. The simulation results indicate that the use of an explicit discretization for the discontinuous part of the input yields numerical chattering. This is not the case when using an implicit discretization. We also provide an example where the use of an explicit discretization of $u^{e q}$ makes the closed-loop system diverge, whereas with the others methods it attains the sliding surface. Further results on discretization performance and stability can be found in the report [18].

\section{REFERENCES}

[1] S. Sarpturk, Y. Istefanopulos, and O. Kaynak, "On the stability of discrete-time sliding mode control systems," Automatic Control, IEEE Transactions on, vol. 32, no. 10, pp. 930-932, 1987.

[2] S. Drakunov and V. Utkin, "On discrete-time sliding modes," in Proc. of IFAC Nonlinear Control System Design Conf., 1989, pp. 273-278.

[3] K. Furuta, "Sliding mode control of a discrete system," Systems \& Control Letters, vol. 14, no. 2, pp. 145-152, 1990.

[4] V. Utkin, "Sliding mode control in discrete-time and difference systems," in Variable Structure and Lyapunov Control, ser. Lecture Notes in Control and Information Sciences. Springer, 1994, vol. 193, pp. 87-107.

[5] W. Gao, Y. Wang, and A. Homaifa, "Discrete-time variable structure control systems," Industrial Electronics, IEEE Transactions on, vol. 42, no. 2, pp. 117-122, 1995. 


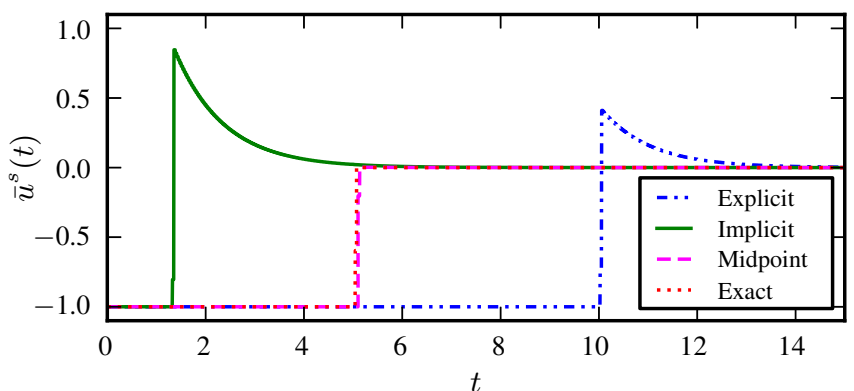

(a) Implicit discretization of $u^{s}$

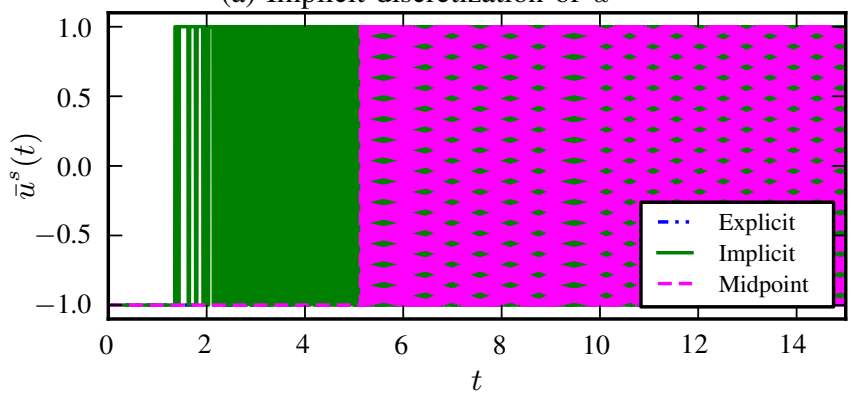

(b) Explicit discretization of $u^{s}$

Fig. 6: Evolution of $\bar{u}^{s}$ for different discretization methods for $u^{e q}$ with $h=0.03 \mathrm{~s}$.

[6] G. Golo and C̆. Milosavljević, "Robust discrete-time chattering free sliding mode control," Systems \& Control Letters, vol. 41, no. 1, pp. 19-28, 2000.

[7] Z. Galias and X. Yu, "Complex discretization behaviors of a simple sliding-mode control system," Circuits and Systems II: Express Briefs, IEEE Transactions on, vol. 53, no. 8, pp. 652-656, 2006

[8] _ _ "Analysis of zero-order holder discretization of two-dimensional sliding-mode control systems," Circuits and Systems II: Express Briefs, IEEE Transactions on, vol. 55, no. 12, pp. 1269-1273, 2008.

[9] B. Wang, X. Yu, and G. Chen, " $\mathrm{ZOH}$ discretization effect on singleinput sliding mode control systems with matched uncertainties," $\mathrm{Au}$ tomatica, vol. 45, no. 1, pp. 118-125, 2009.

[10] V. Acary and B. Brogliato, "Implicit Euler numerical scheme and chattering-free implementation of sliding mode systems," Systems \& Control Letters, vol. 59, no. 5, pp. 284-293, 2010.

[11] V. Acary, B. Brogliato, and Y. Orlov, "Chattering-free digital slidingmode control with state observer and disturbance rejection," Automatic Control, IEEE Transactions on, vol. 57, no. 5, pp. 1087-1101, 2012.

[12] F. Plestan, V. Bregeault, A. Glumineau, Y. Shtessel, and E. Moulay, "Advances in high order and adaptive sliding mode control-theory and applications," in Sliding Modes after the First Decade of the 21 st Century, ser. Lecture Notes in Control and Information Sciences. Springer, 2012, vol. 412, pp. 465-492.

[13] M. Defoort, T. Floquet, A. Kokosy, and W. Perruquetti, "A novel higher order sliding mode control scheme," Systems \& Control Letters, vol. 58, no. 2, pp. 102-108, 2009.

[14] C. Edwards and S. Spurgeon, Sliding Mode Control: Theory and Applications, ser. Systems and Control Book Series. CRC Press, 1998, vol. 7 .

[15] B. Wang, X. Yu, and X. Li, "ZOH discretization effect on higherorder sliding-mode control systems," Industrial Electronics, IEEE Transactions on, vol. 55, no. 11, pp. 4055-4064, 2008.

[16] V. Acary and F. Pérignon, "An introduction to Siconos," INRIA, Rapport Technique RT-0340, 2007. [Online]. Available: http://hal.inria.fr/inria-00162911

[17] J. D. Hunter, "Matplotlib: a 2D graphics environment," Computing in Science \& Engineering, pp. 90-95, 2007.

[18] O. Huber, V. Acary, and B. Brogliato, "Analysis of explicit and implicit discrete-time equivalent-control based sliding mode controllers," pre-print, 2013. [Online]. Available: http://hal.inria.fr/

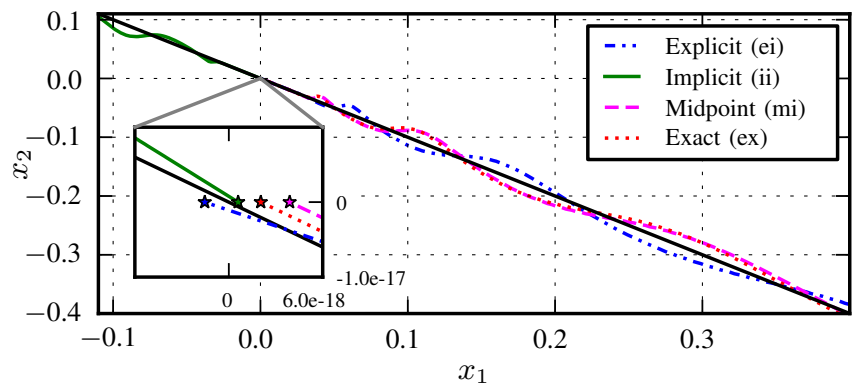

(a) Implicit discretization of $u^{s}$

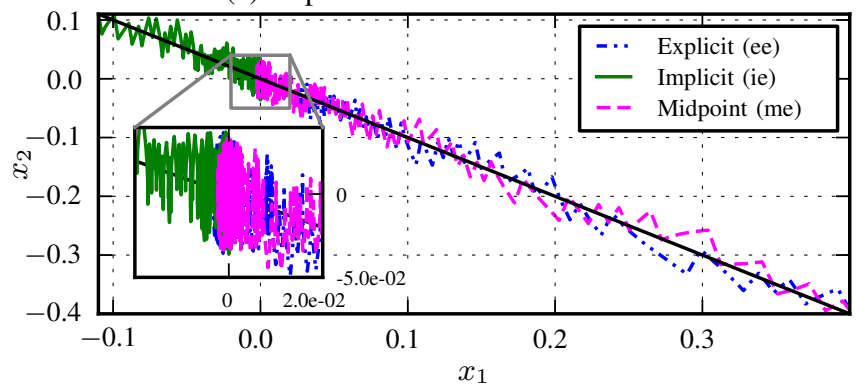

(b) Explicit discretization of $u^{s}$

Fig. 7: Simulations of system (23) using different discretization methods for $u^{e q}$ and $h=0.03 \mathrm{~s}$ (perturbed case).

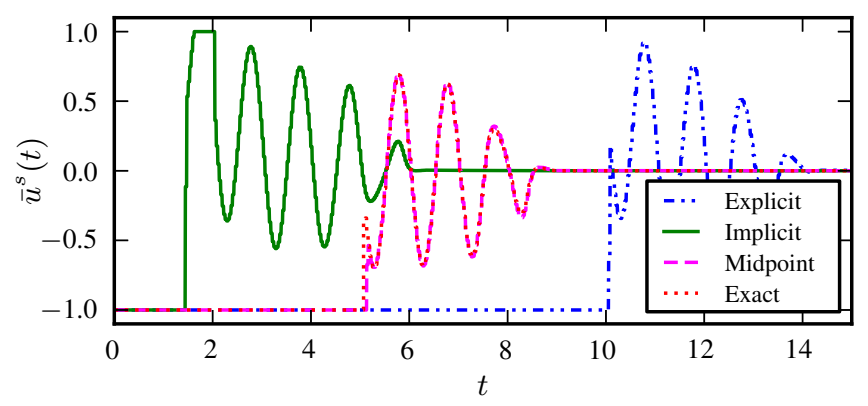

(a) Implicit discretization of $u^{s}$

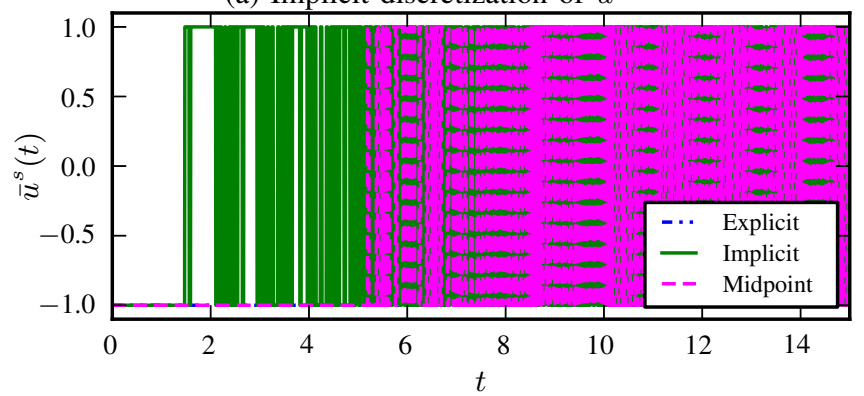

(b) Explicit discretization of $u^{s}$

Fig. 8: Evolution of $\bar{u}^{s}$ for different discretization methods for $u^{e q}$ and $u^{s}, h=0.03 \mathrm{~s}$. (perturbed case) 